\title{
Determination of whether screening tests for chronic atrophic gastritis really has a positive predictive value
}

\author{
FLAVIANO DI PAOLA ${ }^{1 *}$, VALENTINA D'ANGELO $^{2 *}$, FABIANA TATANGELO $^{3}$, VITTORIA BARCHIESI $^{1}$, \\ MARIANNA RIZZO $^{1}$, MONICA CANTILE ${ }^{3}$, GERARDO BOTTI ${ }^{3}$ and ERNESTA CAVALCANTI ${ }^{1}$ \\ ${ }^{1}$ Division of Laboratory Medicine; ${ }^{2}$ Division of Diagnostic and Operative Endoscopy; ${ }^{3}$ Division of Pathology, \\ National Institute of Tumors G. Pascale Foundation, Institute for Hospitalization and Treatment for Scientific Purposes, \\ Naples I-80131, Italy
}

Received April 23, 2014; Accepted January 2, 2015

DOI: $10.3892 / \mathrm{mmr} .2015 .3872$

\begin{abstract}
Intestinal-type gastric adenocarcinomas are preceded by precancerous lesions, which begin with chronic atrophic gastritis. Over the last few years, multiple serological screening techniques have been performed and commercialized for the diagnosis of chronic atrophic gastritis. In the present study, 123 patients were recruited at the International Cancer Institute 'G. Pascale' Foundation (Naples, Italy) to test commercial kits for the serological determination of chronic atrophic gastritis, supported by histological analysis, according to the International Group of Gastroenterologists 'Operative Link for Gastritis Assessment Staging System'. The results revealed a significant discrepancy between serological screening and histological evaluation in $10.6 \%$ of patients, which highlighted the dubious positive predictive value of commercial serological screening kits.
\end{abstract}

\section{Introduction}

Intestinal-type gastric adenocarcinomas are the most common type of gastric tumor, and are preceded by a series of precancerous lesions, beginning with chronic atrophic gastritis, prior to progression to intestinal metaplasia, dysplasia and finally developing into gastric cancer (1). Gastric endoscopy and biopsy collection are frequently used in the diagnosis of chronic atrophic gastritis; however, this procedure is highly invasive. For this reason, numerous experimental and meta-analysis studies have been conducted in order to identify

Correspondence to: Dr Monica Cantile, Division of Pathology, National Institute of Tumors G. Pascale Foundation, Institute for Hospitalization and Treatment for Scientific Purposes, 1 Via M. Semmola, Naples I-80131, Italy

E-mail: monicantile@libero.it

*Contributed equally

Key words: chronic atrophic gastritis, serological test, Operative Link for Gastritis Assessment staging system an effective serological screening method for chronic atrophic gastritis (2-6).

However, the level of variation in the results of these studies have made it difficult to identify a standardized method for the effective diagnosis of chronic atrophic gastritis.

Currently, commercially available serological screening tests identify four main molecular markers: Pepsinogen I (PGI, 30-160 mg/l), whose concentration levels in the blood reflect the structure and function of the mucosa of the gastric body (corpus); pepsinogen II (PGII, 3-15 g/l), whose concentration levels are associated with the structure and function of the mucosa of the stomach; gastrin-17b (G17b, 1-7 pmol/l), whose concentration levels reflect the structure and function of the antrum mucosa of the stomach and Helicobacter pylori (HPAbG, <30 EIU), which is responsible for gastric mucosal atrophy and dysfunction.

The evaluation of the ratio of PGI:PGII, and the detected combinations of these markers should provide adequate and complete information regarding the structure and function of the gastric mucosa (7). The present study therefore aimed to evaluate the positive predictive value of such commercial serological screening kits via comparison with data from histological evaluation.

\section{Materials and methods}

Patients. In the present study, 123 patients (mean age $53 \pm 14$ years), who gave their informed consent and had been referred by their general practitioner for endoscopy, were enrolled at the Institute for Cancer Cure and Research, the International Cancer Institute 'G. Pascale' Foundation (Naples, Italy). They donated blood and underwent an endoscopy. A total of 13 patients (six females and seven males; mean age $51 \pm 16$ years) were selected due to discrepancies observed between serological and histological analyses. The study was approved by the ethics committee of INT Fondazione Pascale.

Determination of biological marker expression. All blood samples were tested for antibodies against $H$. pylori, PGI, PGII and G17b using a GastroPanel ${ }^{\circledR}$ commercial ELISA kit (cod. 601300; Biohit HealthCare, Helsinki, Finland) assay. The final report contained the results of biological marker 


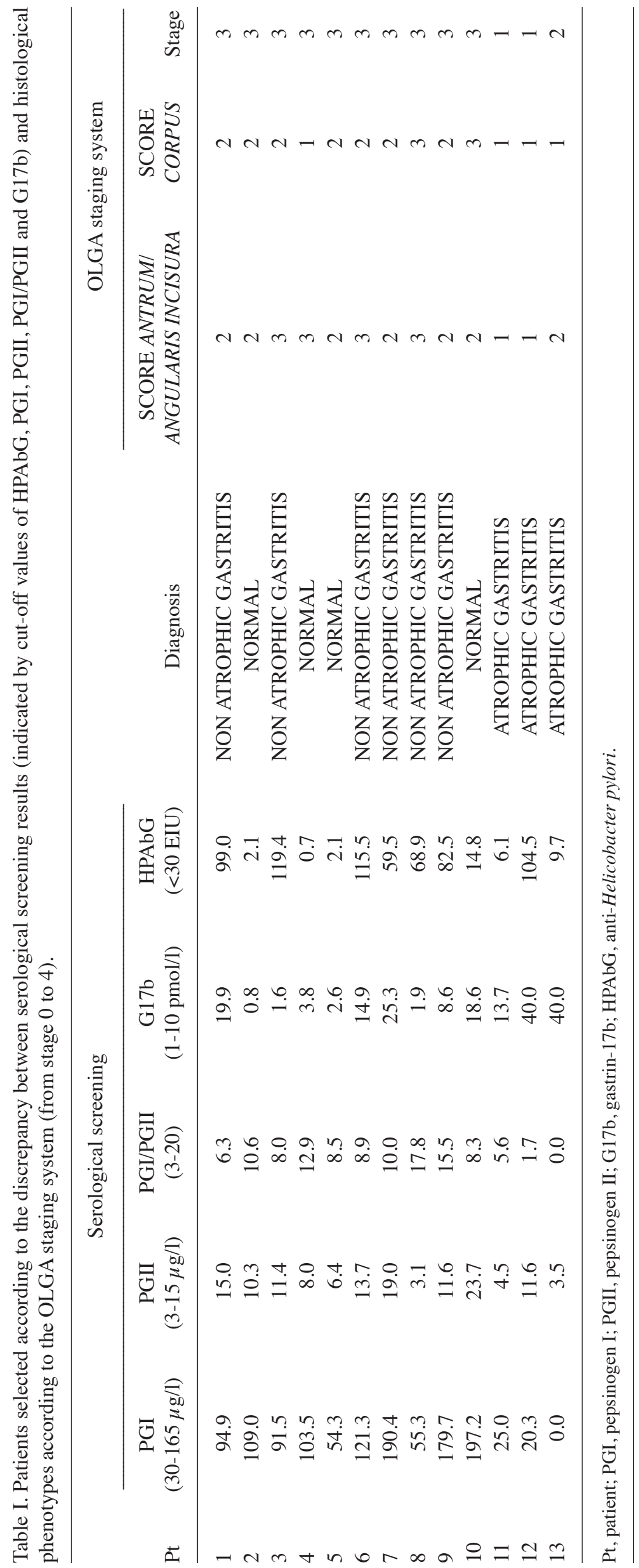



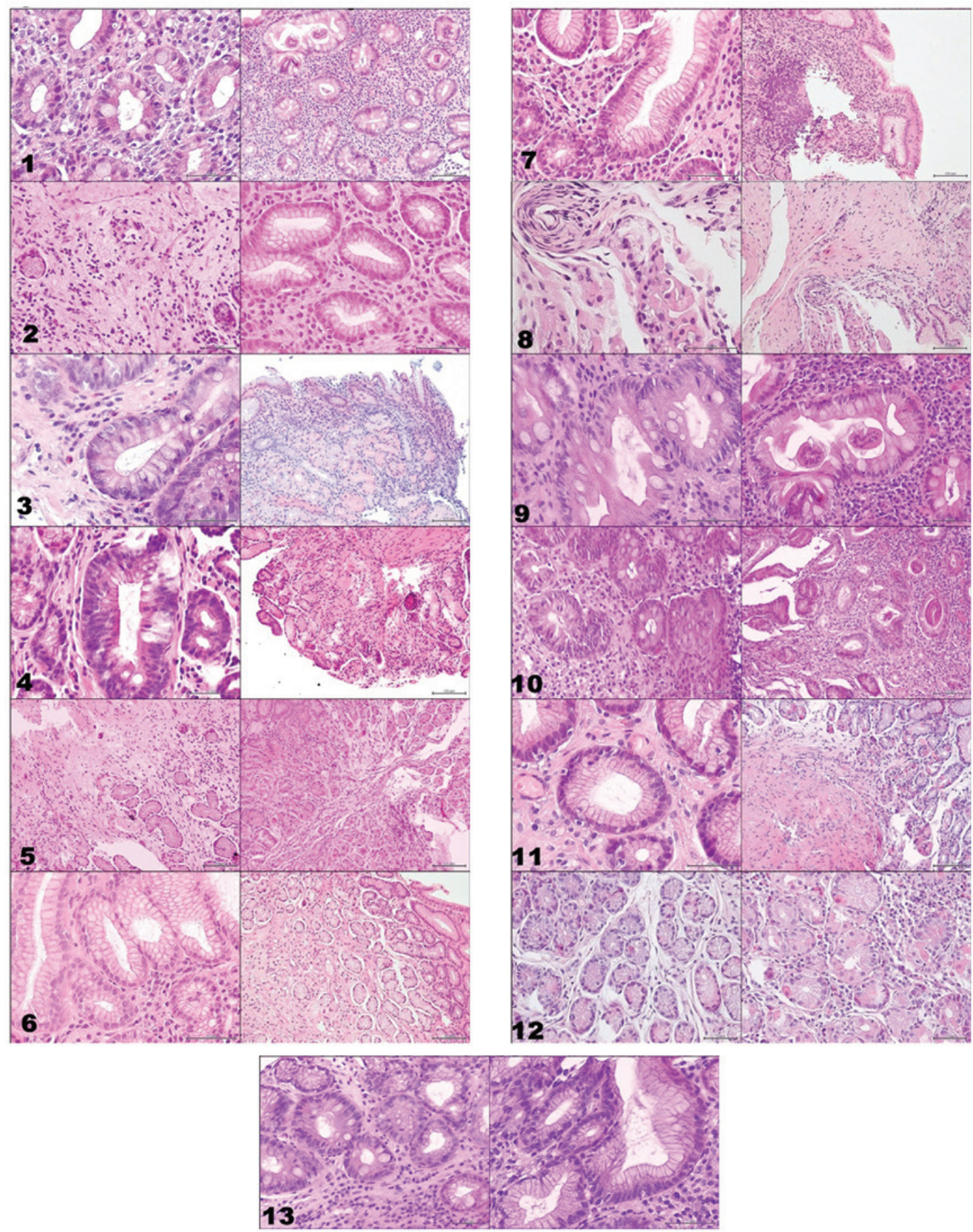

Figure 1. Histology of the antrum (left) and the corpus (right) of the thirteen discrepant patients identified. Magnification: 1) Left x60, right 20x; 2) left x60, right $\mathrm{x} 60 ; 3$ ) left $\mathrm{x} 60$, right $\mathrm{x} 40 ; 4)$ left $\mathrm{x} 60$, right $\mathrm{x} 40 ; 5$ ) left $\mathrm{x} 40$, right $\mathrm{x} 40 ; 6$ ) left $\mathrm{x} 60$, right $\mathrm{x} 60 ; 7$ ) left $\mathrm{x} 60$, right $\mathrm{x} 40 ; 8)$ left $\mathrm{x} 60$, right $\mathrm{x} 40 ; 9)$ left $\mathrm{x} 60$, right $x 60 ; 10$ ) left $x 60$, right $x 40 ; 11)$ left $x 60$, right $x 40 ; 12$ ) left $x 60$, right $x 60 ; 13)$ left $x 40$, right $x 60$.

expression levels, including the cut-off values outlined in Table I.

Histological examination. The stomach mucosa was evaluated using routine methods, and biopsies were taken from the corpus, antrum and angularis incisura for histological examination. Formalin-fixed, paraffin-embedded sections $(5 \mu \mathrm{m})$, were stained with hematoxylin/eosin by automated instruments (Stainer AUS124; Bio Optica, Milan, Italy) and the results were interpreted using a light microscope, observing a minimum of 10 fields (Leica DM 1000; Leica Microsystems GmbH, Heidelberg, Germany). Histological parameters were outlined according to the 'Operative Link for Gastritis Assessment (OLGA) Staging System', which was defined by an international group of gastroenterologists and pathologists. The OLGA staging system defines the histological phenotypes of gastritis according to a scale of progressively increasing risk of gastric cancer, from lowest (stage 0) to highest (stage 4) (8). To minimize disagreement amongst pathological diagnoses of chronic atrophic gastritis, 
two independent reviews of each biopsy were required from two pathologists, culminating in a final consensus diagnosis for each patient.

\section{Results}

Intestinal-type gastric adenocarcinomas are preceded by a series of precancerous lesions, initiating with chronic atrophic gastritis (9). The development of techniques to effectively perturb the progression of such diseases represents a potentially significant public health benefit, reducing the associated economic burden of medical expenses and relieving the suffering of patients.

In the present study, 123 patients were enrolled to evaluate a commercial kit for the serological determination of chronic atrophic gastritis, compared with histological analysis.

The results indicated a significant discrepancy between the results of serological screening and histological evaluation amongst 13 of the 123 patients (10.6\%; Table I and Fig. 1).

Ten patients $(8.1 \%)$ were identified to be negative following serological screening (normal phenotype or non-atrophic gastritis) and positive at histology (OLGA stage $\geq 3$ ) (Table I and Fig. 1). Amongst these patients, four exhibited a normal serological phenotype and six demonstrated non-atrophic gastritis. By contrast, three patients $(2.4 \%)$ revealed a positive serological screening indicative of atrophic gastritis, while the histological examination was considered negative (OLGA stage $\leq 2$ ). One case exhibited histology indicating stage 2 atrophic gastritis and two cases exhibited histology indicating stage 1 atrophic gastritis (Table I and Fig. 1).

\section{Discussion}

Numerous studies have demonstrated the sensitivity and specificity of serological screening tests for chronic atrophic gastritis, determining a range of cut-off values used in commercial kits (10).

However, the significant levels of variation described in experimental studies, despite numerous validation studies (11), may also be present in standardized commercial methods. This may be due to discrepancies in population characteristics, for example country of origin or patient selection criteria, and also to variations in the cut-off values used (11).

In particular, in the present study, the patients evaluated represented a select group, often exhibiting clinical disease, which represents a common limitation amongst studies of various screening tests (11-13).

Studies have yielded conflicting results regarding the use of the biomarkers evaluated in the present study. For example, serological evaluation of PGI, PGII, the ratio of PGI and
PGII and serum gastrin revealed that only the ratio of PGI to PGII was reliable, demonstrating a specificity of $87 \%$ and a sensitivity of $77 \%$ for the detection of chronic atrophic gastritis (12). However, even in this example, the study was limited by the recruitment of a patient cohort presenting with dyspeptic symptoms (for endoscopy), which may increase the probability of gastric atrophy identification. Furthermore, in alternative studies conducted on selected populations, the positive predictive value of serum PG for gastric cancer was demonstrated to be low (13).

In conclusion, although existing commercial tests for chronic atrophic gastritis are suggested to be highly sensitive and specific, the results of the present study revealed that they may not have the positive predictive value that has been previously reported.

\section{References}

1. Dicken BJ, Bigam DL, Cass C, et al: Gastric adenocarcinoma review and considerations for future directions. Ann Surg 241: 27-39, 2005.

2. Thijs JC, van Zwet AA, Thijs WJ, et al: Diagnostic tests for Helicobacter pylori: a prospective evaluation of their accuracy, without selecting a single test as the gold standard. Am J Gastroenterol 91: 2125-2129, 1996.

3. Mårdh E, Mårdh S, Mårdh B and Borch K: Diagnosis of gastritis by means of a combination of serological analyses. Clin Chim Acta 320: 17-27, 2002

4. Ricci C, Vakil N, Rugge M, et al: Serological markers for gastric atrophy in asymptomatic patients infected with Helicobacter pylori. Am J Gastroenterol 99: 1910-1915, 2004.

5. Mizuno S, Miki I, Ishida T, et al: Prescreening of a high-risk group for gastric cancer by serologically determined Helicobacter pylori infection and atrophic gastritis. Dig Dis Sci 55: 3132-3137, 2010

6. Bornschein J, Selgrad M, Wex T, et al: Serological assessment of gastric mucosal atrophy in gastric cancer. BMC Gastroenterol 12: $10,2012$.

7. Miki K, Morita M, Sasajima M, et al: Usefulness of gastric cancer screening using the serum pepsinogen test method. Am J Gastroenterol 98: 735-739, 2003.

8. Rugge M, Genta RM and OLGA Group: Staging gastritis: an international proposal. Gastroenterology 129: 1807-1808, 2005.

9. Correa P and Piazuelo MB: Helicobacter pylori infection and gastric adenocarcinoma. US Gastroenterol Hepatol Rev 7: 59-64, 2011.

10. Nasrollahzadeh D, Aghcheli K, Sotoudeh M, et al: Accuracy and cut-off values of pepsinogens I, II and gastrin 17 for diagnosis of gastric fundic atrophy: influence of gastritis. PLoS One 6: P1, 2011.

11. Haj-Sheykholeslami A, Rakhshani N, Amirzargar A, et al: Serum pepsinogen I, pepsinogen II and gastrin 17 in relatives of gastric cancer atrophy patients: comparative study with type and severity of gastritis. Clin Gastroenterol Hepatol 6: 174-179, 2008.

12. Broutet N, Plebani M, Sakarovitch C, et al: Pepsinogen A, pepsinogen $\mathrm{C}$ and gastrin as markers of atrophic chronic gastritis in European dyspeptics. Br J Cancer 88: 1239-1247, 2003.

13. Miki K, Morita M, Sasajima M, et al: Usefulness of gastric cancer screening using the serum pepsinogen test method. Am J Gastroenterol 98: 735-739, 2003. 